\title{
Development of Metal-Organic Framework (MOF) Sensors for Landmine Detection
}

\author{
Lisa H. Humphreys ${ }^{1}$, Ian J. Wilson ${ }^{1}$, Daniel McAteer ${ }^{1}$, Jean-François Pons ${ }^{1}$ \\ ${ }^{1}$ Centre for Defence Chemistry, Cranfield University, \\ Defence Academy of the United Kingdom, Shrivenham, SN6 8LA, UK \\ I.humphreys@cranfield.ac.uk
}

\begin{abstract}
Rural populations in Colombia are significantly affected by anti-personnel mines used by revolutionary armed forces. These mines typically contain ammonium nitrate (AN) based explosive compositions. The international community is committed to tackling the danger posed by landmines and other improvised explosive devices (IEDs). This involves the removal of explosive materials installed in areas of conflict. Current technologies used for humanitarian demining can be separated into three distinct categories; metal detection, mechanical clearance, and detection using trained animals [1]. Metal detection is deemed to be most effective but as plastic-bodied IEDs are now also in use other detection approaches must be sought after. Cranfield University, Universidad Nacional de Colombia and Oxford University have proposed the development of a device capable of detecting AN based IEDs using an optoelectronic sensor. The goal of the project is to establish the scientific validity of the new sensing method for the detection of explosive materials found in landmines and IEDs, and to demonstrate its usefulness in locating buried explosive devices. This will entail optimising the sensitivity and selectivity of the sensor. A systematic series of MOFs comprising transition metal acetates/chlorides and terephthalic acid derivatives as linkers has been synthesised and structurally characterised. Cobalt and copper based salts have been paired with numerous organic ligands to create MOFs which have been responsive to concentrations of $\sim 100 \mathrm{ppm}$ ammonia in aqueous conditions. The developed MOFs have also been assessed for their response to ammonia in the gas phase and at representative concentrations throughout the project.
\end{abstract}

Key words: metal-organic frameworks (MOFs), sensors, detection, improvised explosive devices (IEDs), ammonia.

\begin{abstract}
Introduction
Improvised explosive devices (IEDs), such as antipersonnel mines, have claimed many lives and have been the cause of countless life changing injuries. Colombia has one of the highest numbers of antipersonnel mines in the world [1]. IED accidents have not only affected military personnel but also the civilian population, many of whom have been children. To aid humanitarian demining programs, improved sensors are required, as currently available commercial detection systems cannot identify IEDs effectively due to the nature of the ingredients used within them. They comprise a mixture of ammonium nitrate (AN) and fuel oil (FO) both of which are ubiquitous and therefore prone to provide false positive results.
\end{abstract}

The main advantages of electrochemical sensors are that they are light, cheap, and robust. For the sensor to be effective it also needs to be highly selective and only respond to a prescribed analyte (in particular AN). An alternative approach is based on optoelectronic sensor materials, including luminescence from polymers or metal-organic frameworks (MOFs). MOFs are currently being investigated for various sensor applications. These are crystalline materials which contain metal ions/clusters and bridging organic ligands that assemble to form highly porous 3D structures. The open metal sites and pores within can be used to encapsulate target molecules such as ammonia which evolves from AN. This can trigger a detectable response, such as a visible colour change, even when exposed to low ppm levels of the target species.

\section{Experimental}

Several MOFs have been designed based on an initial literature survey of materials which are known to interact with ammonia and produce a 


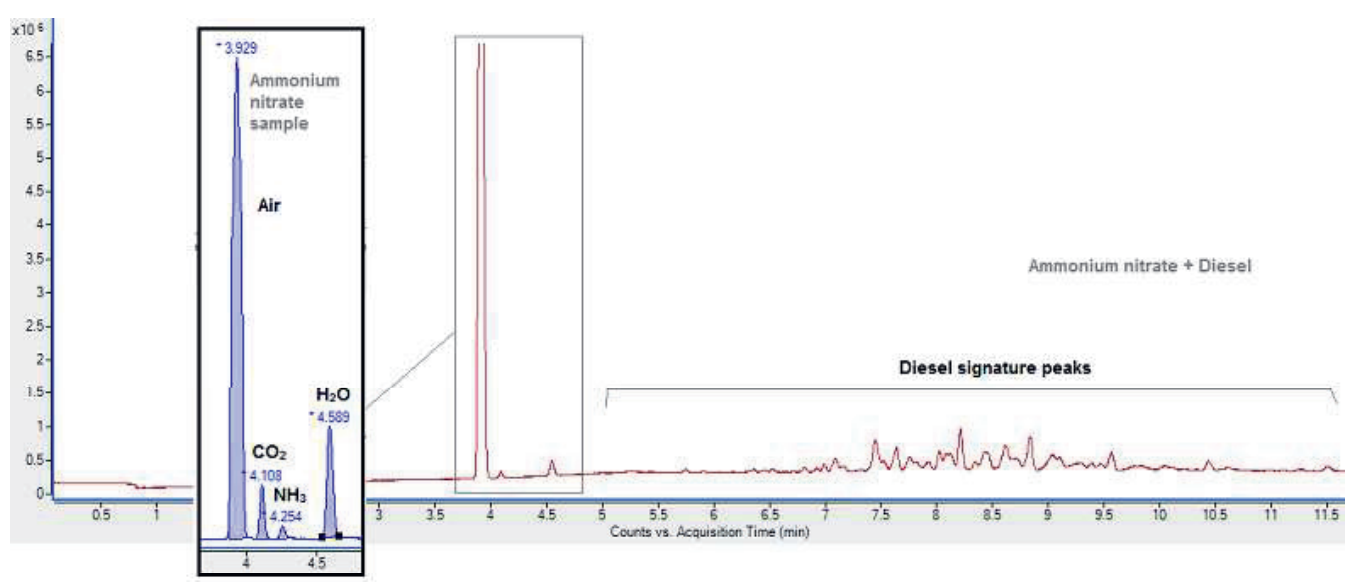

Fig.1. Example GCMS result associated with ANFO.

detectable response [2]. Transition metal acetates and chlorides ( $\mathrm{Co}, \mathrm{Cu}, \mathrm{Zn}$ and $\mathrm{Zr}$ ) and various organic linkers have been combined through both a room-temperature synthesis and a low pressure solvothermal synthesis. The MOFs are being characterised by X-ray diffraction and thermal analysis whilst their responsiveness to the analyte is quantified.

\section{Results and Discussion}

Early analyses suggested ammonia as a viable vapour analyte for detection. The specific amount liberated was evaluated by gas chromatography mass spectrometry (GCMS) (Fig. 1). The level of $\mathrm{NO}_{x}$ generated was assessed by chemiluminescence, however, the values fall within the $\mathrm{ppb}$ range and based on the higher environmental level of $\mathrm{NO}_{x}(\mathrm{NO}$ and $\mathrm{NO}_{2}$ ) present in air this analyte was deprioritised as an analyte of interest. Co BDC and $\mathrm{Co} \mathrm{NH}_{2} \mathrm{BDC}$ have shown great promise as MOF sensors. They change from pink to bluegreen as the ammonia concentration increases to $>100 \mathrm{ppm}$. The lower limit of detection reached to date for aqueous ammonia is 100 300 ppm (Fig. 2 and Fig. 3).

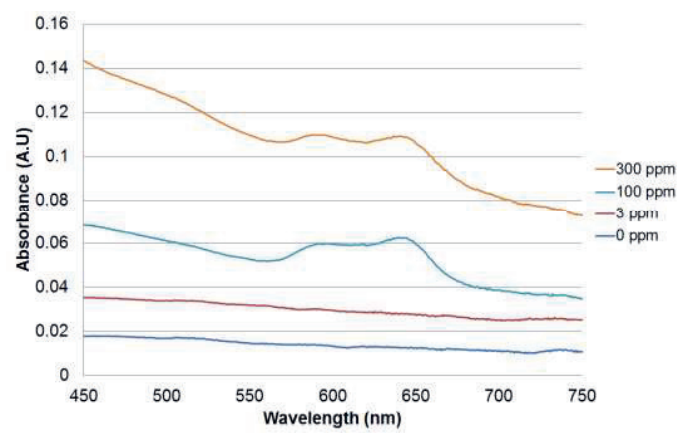

Fig.2. UV-Vis spectra of Co BDC MOF responses to aqueous ammonia $(0-300 \mathrm{ppm})$

The drivers for the specific interaction between ammonia and the ligand are being assessed by modifying the substitution pattern on the ligand to evaluate the effect on the response. These ligands are not commercially available therefore, they are being synthesised at Cranfield University.

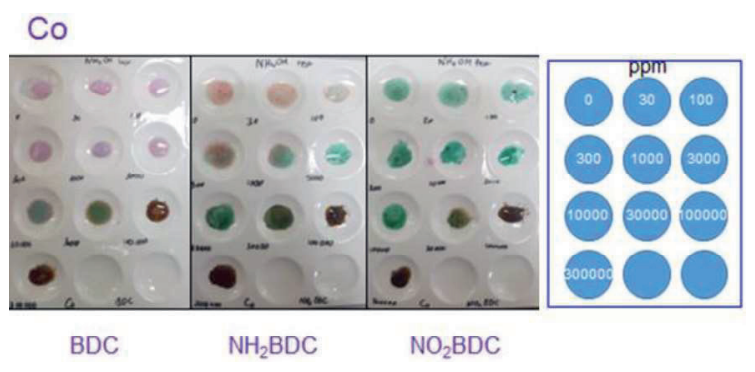

Fig.3. Co based MOF responses to aqueous ammonia (0 - 300,000 ppm)

\section{Conclusion}

Initial MOF candidates, especially those which include $\mathrm{Co}$ and $\mathrm{Cu}$, have shown promising colorimetric changes in response to ammonia with visual detection as low as $100 \mathrm{ppm}$. Current and future work will focus on the development of new ligands and new MOFs to increase their sensitivity and selectivity for the detection systems.

\section{Acknowledgements}

Research supported by the British Council Newton Fund, Grant I.D. IL216431495. Thank you to Yara UK for the complimentary supply of fertilisers.

\section{References}

[1] P. A. Prada, M. C. Rodríguez, Demining Dogs in Colombia - A Review of Operational Challenges, Chemical Perspectives, and Practical Implications, Science and Justice, 56, 269-277 (2016); doi: 10.1016/j.scijus.2016.03.002

[2] K. Vikrant, V. Kumar, K. Kim, D. Kukkar, Metalorganic frameworks (MOFs): potential and challenges for capture and abatement of ammonia, Journal of Materials Chemistry A, 5, 22877-22896 (2017); doi: 10.1039/c7ta07847a 